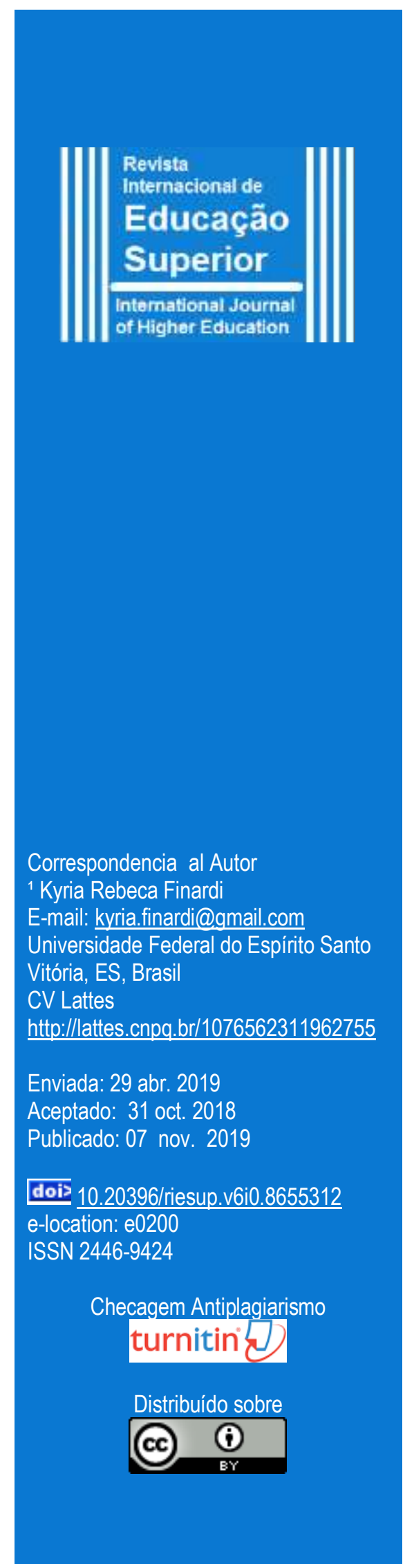

\title{
Pensando la Internacionalización (Crítica) de la Enseñanza Superior Brasileña
}

Kyria Rebeca Finardi ${ }^{1}$ http://orcid.org/0000-0001-7983-2165

Felipe Furtado Guimarães ${ }^{2}$ (id https://orcid.org/0000-0001-6184-3691

Ana Rachel Mendes ${ }^{3}$ (D) https://orcid.org/0000-0002-4359-1109

1,2,3 Universidade Federal do Espírito Santo

\section{RESUMEN}

Este estudio ofrece un espacio para pensar la internacionalización de las instituciones de enseñanza superior brasileñas de forma más crítica a partir de un meta-análisis de los estudios de un grupo de investigación que aborda cuestiones como programas de internacionalización y políticas lingüísticas y de internacionalización en ese contexto. Un hallazgo del meta-análisis sugiere una brecha (y desajuste) de principios y políticas para guiar el proceso de internacionalización de forma crítica en Brasil. La internacionalización crítica se aborda teniendo en cuenta el papel de programas y políticas lingüísticas y de internacionalización bien como a partir del reconocimiento de la mercantilización de la educación y de los medios de contrarrestarla. Para tanto, se sugiere la desconstrucción del imaginario jerárquico de dominación intelectual y cultural y la interacción solidaria entre las instituciones de los países periféricos para fortalecer el carácter de bien público de la enseñanza superior.

\section{PALABRAS CLAVE}

Internacionalización. Políticas lingüísticas. Internacionalización crítica.

Enseñanza superior. 


\title{
Reflecting on Brazilian Higher Education (Critical) Internationalization
}

\begin{abstract}
This study offers a space to think about the internationalization of Brazilian higher education institutions in a more critical way based on a meta-analysis of the studies of a research group that addresses issues such as internationalization programs, language policies and internationalization in that context. Findings of the metaanalysis suggest a gap (and mismatch) of principles and policies to critically guide the process of internationalization in Brazil. This study approaches critical internationalization considering the role of language and internationalization programs and policies, as well as the recognition of the commodification of education and the means of counteracting it. Therefore, it is suggested the deconstruction of the hierarchical imaginary of intellectual and cultural domination, and the solidary interaction among the institutions of the peripheral countries to strengthen the understanding of higher education as a public good.
\end{abstract}

\section{KEYWORDS}

Internationalization. Language policies. Critical internationalization. Higher education.

\section{Pensando na Internacionalização (Crítica) do Ensino Superior Brasileiro}

\section{RESUMO}

Este estudo oferece um espaço para pensar a internacionalização das instituições de ensino superior brasileiras de maneira mais crítica, com base em uma metanálise dos estudos de um grupo de pesquisa que aborda questões como programas de internacionalização e políticas lingüísticas e de internacionalização. contexto. Um achado de meta-análise sugere uma lacuna (e incompatibilidade) de princípios e políticas para guiar o processo de internacionalização de forma crítica no Brasil. A internacionalização crítica é abordada levando em consideração o papel dos programas e políticas de linguística e internacionalização, bem como a partir do reconhecimento da mercantilização da educação e dos meios para combatê-la. Portanto, sugere-se a desconstrução do imaginário hierárquico de dominação intelectual e cultural e a interação solidária entre as instituições dos países periféricos para fortalecer o bem público do ensino superior.

\section{PALAVRAS-CHAVE}

Internacionalização. Políticas lingüísticas. Internacionalização crítica. Ensino superior. 


\section{Introducción}

El Informe sobre Desarrollo Humano de 1999 (UNITED NATIONS, 1999) afirma que el colapso del espacio, del tiempo y de las fronteras promovido por la globalización, crea una aldea global, pero de la cual ni todos pueden ser ciudadanos. En ese escenario, mientras la élite global y profesional encuentran fronteras bajas o, por veces, inexistentes, millones de personas tienen que enfrentar fronteras altas y persistentes. Igualmente, la globalización ha promovido un aumento en los flujos de tecnología, conocimiento, personas, valores e ideas, promoviendo el fenómeno de la internacionalización de la enseñanza superior con beneficios exclusivos a algunos, pero no disponibles a todos de forma igualitaria (VAVRUS; PEKOL, 2015).

Existe una estrecha relación entre los conceptos de internacionalización, globalización y regionalización de la enseñanza superior (LÓPEZ; SANMARTÍN, 2004). Con relación a los efectos de la globalización/internacionalización de la enseñanza superior, Knight (2008) afirma que la internacionalización está cambiando el mundo de la enseñanza superior y la globalización está cambiando el mundo de la internacionalización. Una vez que ambos afectan la forma en que los ciudadanos ven las fronteras, la internacionalización se ha convertido en un aspecto importante en la vida de las personas, actuando al mismo tiempo como consecuencia y como agente de la globalización.

Brandenburg y De Wit (2010) comentan que la internacionalización generalmente es considerada como algo positivo y la globalización como algo negativo. Según esos autores, el antagonismo construido entre internacionalización y globalización ignora el hecho de que las actividades que están más relacionadas con el concepto de globalización, esto es, la enseñanza superior como un producto comercializable, se llevan a cabo cada vez más por la internacionalización.

López y Sanmartín (2004) discuten la globalización, internacionalización y regionalización de la enseñanza superior partiendo de las siguientes características que aproximan/distancian esos procesos:

Cuadro 1. Características de la Internacionalización y Globalización

\begin{tabular}{|l|l|}
\hline \multicolumn{1}{|c|}{ Internacionalización } & \multicolumn{1}{c|}{ Globalización } \\
\hline $\begin{array}{l}\text { Asume un mundo de naciones con fronteras geo-políticas } \\
\text { claras que se pretenden superar con actividades tales } \\
\text { como el intercambio de estudiantes, de profesorado, la } \\
\text { colaboración en la investigación, etc. }\end{array}$ & Es totalmente contraria a un mundo de Estados. \\
$\begin{array}{l}\text { Se apoya en las altas esferas de la diplomacia y de la } \\
\text { cultura. Por ejemplo, la movilidad de estudiantes ha sido } \\
\text { promocionada de forma frecuente por convenios } \\
\text { bilaterales entre países que compartían vínculos } \\
\text { históricos. Por tanto, es más fácil de controlar o regular. }\end{array}$ & $\begin{array}{l}\text { Se apoya en el consumo de masas y en el } \\
\text { capitalismo mundial. Por ejemplo, a través de } \\
\text { internet es el alumno quien decide que universidad } \\
\text { desea visitar virtualmente. Difícilmente } \\
\text { controlable. }\end{array}$ \\
$\begin{array}{l}\text { Tiende a reproducir, e incluso a legitimar, la jerarquía y la } \\
\text { cientaca la jerarquía y la hegemonía tradicionales. }\end{array}$ \\
\hline
\end{tabular}


hegemonía. Así, determinados países que fueran los centros de imperios de siglos pasados, atraen a los alumnos procedentes de antiguas colonias.

Su motor debería ser algo más altruista; la expansión del conocimiento y la investigación, así como la crítica constructiva a través de la cooperación.

No es algo nuevo, sino una faceta con la que la universidad se ha encontrado tradicionalmente a gusto.
Por ejemplo, una universidad sin tradición internacional y sin demasiados medios económicos puede llegar a otros estudiantes a través de la red.

Su principal motor podría considerarse el beneficio y la creencia en un mercado global único que se consigue fundamentalmente a través de la competitividad.

Se trata de algo novedoso y dinámico. Podríamos decir que surge a principios del siglo XIX, o incluso antes, asociada al nacimiento de la revolución industrial.

Fuente: Adaptado de López y Sanmartín (2004).

Esos autores concluyen que los procesos de internacionalización, globalización y regionalización de la enseñanza superior cobran cada vez mayor importancia de los agentes implicados en la educación. Sin embargo, la situación de desigualdad entre los diferentes sistemas de enseñanza superior respecto de la movilidad académica (por ejemplo, LIMA; MARANHÃO, 2009 en el caso de Brasil), es discutida por López y Sanmartín (2004) a través de un análisis de cluster de los flujos de estudiantes internacionales de los países de la Organización para la Cooperación y Desarrollo Económico (OCDE). Dicho análisis sugiere que la movilidad tiende a producirse entre áreas geográficas habitualmente próximas, que mantienen vínculos de carácter político, histórico y cultural. Tales vínculos, según esos autores, facilitan el establecimiento de acuerdos de cooperación permitiendo incorporar el factor de regionalización al proceso de internacionalización de la enseñanza superior. Desde ese enfoque, se puede deducir que la regionalización puede promover la superación de barreras, constituyéndose un motor de la internacionalización.

Para De Wit (2011), las dinámicas cambiantes en la internacionalización de la enseñanza superior se reflejan tanto en los significados de la internacionalización y la globalización como en sus bases. Aunque el autor reconozca que siempre ha habido muchos términos diferentes utilizados en relación con la internacionalización de la enseñanza superior (DE WIT, 2002; KNIGHT, 2008), en los últimos 20 años, se ha podido observar la aparición de un nuevo grupo de términos que no habían estado presentes de forma activa en el debate sobre la internacionalización de la enseñanza superior. Estos nuevos términos se relacionan mucho más con la oferta transfronteriza de enseñanza y se presentan como una consecuencia del impacto de la globalización en la enseñanza superior: enseñanza sin fronteras, enseñanza a través de fronteras, enseñanza global, enseñanza en el exterior y el comercio internacional en los servicios de enseñanza.

Gonzáles (2007) discute la diferencia entre internacionalización y educación fronteriza con base en referencias del Documento de Orientación de la Organización de las Naciones Unidas para la Educación, la Ciencia y la Cultura (UNESCO). Según ese documento, la mundialización y los cambios recientes en la prestación internacional de servicios de educación superior, han dado origen a nuevas expresiones entre las cuales están "educación sin fronteras", "educación transnacional", "educación a través de las fronteras", o "educación 
transfronteriza". La educación sin fronteras se refiere a la ausencia de las fronteras conceptuales mientras que las otras expresiones, en realidad enfatizan la existencia de fronteras. Ambos enfoques reflejan la realidad actual y se ligan al concepto de internacionalización, globalización y regionalización de la enseñanza superior. El crecimiento sin precedentes de la educación a distancia, y con apoyo de la internet, sugiere el desaparecimiento de las fronteras geográficas. Con todo, las fronteras adquieren mayor importancia cuando la atención se concentra en cuestiones como el flujo de la movilidad académica, el acceso y la financiación de la enseñanza superior.

Concordamos en parte con López y Sanmartín (2004), De Wit (2011) y Gonzáles (2007) en que la internacionalización/regionalización de la enseñanza superior con el uso de internet puede promover el desaparecimiento de fronteras. Sin embargo, pensamos que los procesos de regionalización/internacionalización de la enseñanza superior deben considerar otros factores, como el uso de lenguas y las relaciones de poder subyacentes en esos procesos, como también las relaciones entre instituciones de enseñanza superior/países de diferentes puntos geográficos y geopolíticos del mundo. En ese sentido, este trabajo tiene como objetivo reflexionar sobre el uso de lenguas y las relaciones de poder existentes en el proceso de regionalización/internacionalización de la enseñanza superior en Brasil, esto con la finalidad de proponer una visión más crítica y sustentable de esos procesos.

\section{Internacionalización}

Según Altbach y Knight (2006), la internacionalización de la enseñanza superior ha cambiado mucho en las últimas décadas con el incremento significativo de su volumen, alcance y complejidad. Esos autores entienden que la internacionalización, y más específicamente la educación comercial transfronteriza, es un negocio atractivo que implica ganancias considerables.

Knight (2003, p.2, nuestra traducción) define la internacionalización como "el proceso de integración de una dimensión internacional, intercultural o global en el propósito, en las funciones o en la entrega de la educación post-secundaria". La internacionalización abarca actividades como la movilidad académica, acuerdos entre instituciones entre otras actividades, como las descritas por Amorim y Finardi (2017), Finardi, Santos y Guimarães (2016) y Finardi y Guimarães (2017), en el caso de la internacionalización en un contexto específico en Brasil. Knight (2003) sugiere que, aunque la internacionalización generalmente implique algún tipo de movilidad o intercambio académico, no debe limitarse solamente a esto, sino que debe entenderse como una forma de mejorar la misión tripartita de las universidades (proporcionar enseñanza, investigación y servicios).

Otro aspecto de la internacionalización a ser considerado, es cómo una institución integra los aspectos descritos por Knight (2003) en su definición de internacionalización. Nos referimos al término "Internacionalización en Casa" ( $\mathrm{IeC}$ ), que puede ser visto como "cualquier actividad relacionada internacionalmente con la excepción de la movilidad 
estudiantil saliente" (NILSSON, 2003, p.31, nuestra traducción). Beelen y Jones (2015, p.69, nuestra traducción) presentaron una definición más reciente para $\mathrm{IeC}$, como siendo una "integración intencional de las dimensiones internacionales e interculturales en el currículo formal e informal para todos los estudiantes dentro de entornos de aprendizaje doméstico".

Hudzik (2011, p. 10, nuestra traducción) sugiere pensar la internacionalización como un proceso integral, tal como:

un compromiso confirmado a través de la acción, para infundir perspectivas internacionales y comparativas a través de las misiones de enseñanza, investigación y servicio de la enseñanza superior. Da forma al espíritu y a los valores institucionales y afecta a toda la empresa de la enseñanza superior. Es esencial que sea abrazado por el liderazgo institucional, el gobierno, la facultad, los estudiantes y todas las unidades de servicio y apoyo académico. Es un imperativo institucional, no solo una posibilidad deseada.

Por otro lado, se puede reconocer en este imperativo de internacionalización, un imperialismo nuevo, sutil y benevolente en el sistema de educación terciaria en la que los países hegemónicos ${ }^{1}$ buscan implementar su estándar de educación e infundir sus valores en los países periféricos, expandiendo su dominio intelectual, cultural y financiero (LEITE; GENRO, 2012).

La intencional implementación de una política internacional de educación, de un estándar europeo, con el objetivo de alcanzar el mundo en su totalidad, incluye a Latinoamérica y el Caribe; expresa el ejercicio del poder europeo en una búsqueda para alcanzar intereses capitalistas a través de corazones y mentes.

El imperialismo benevolente irradia su proceso de dominación y explotación, a través de una perspectiva que hace posible ampliar la forma de dominación con un mínimo de coacción. La hegemonía en esta forma de imperialismo se hace por la promoción de un modo de vida, formas de pensar, actuar y consumir en los moldes de la lógica del mercado europeo. La educación, en ese sentido, tiene un papel fundamental en la socialización de normas y principios compatibles con ese mundo existente. (LEITE; GENRO, 2012, p. 770, nuestra traducción)

La internacionalización se puede utilizar como una plataforma para promover la visibilidad mundial de las instituciones de enseñanza superior. Según Finardi y Ortiz (2015), esta promoción puede estar "motivada económicamente" una vez que las universidades esperan que se financien, al menos en parte, con los pagos realizados por alumnos extranjeros. De hecho, muchos autores (entre ellos, LEITE; GENRO, 2012; ANDREOTTI et al., 2016; PICCIN; FINARDI; 2019) señalan las lógicas de mercantilización de la globalización hegemónica que han entrado en las universidades, llevando la enseñanza superior a ser tratada como un servicio a ser vendido y comprado y no más como un bien

\footnotetext{
${ }^{1}$ En este estudio, los países hegemónicos y los países periféricos se definen de acuerdo con las ideas presentadas por Santos (2011). Este autor describió el escenario internacional que indica que los países hegemónicos son los que producen conocimiento y "empujan" dicho conocimiento para que sea adoptado en países periféricos, sin las adaptaciones necesarias en contextos locales. Estas ideas se ampliaron para describir un "norte global" y un "sur global", conceptos que explicamos más adelante.
} 
público, aumentando así la brecha entre los países hegemónicos (del Norte) que venden esos productos y los periféricos (del Sur) que los compran y consumen.

Por esa lógica, una forma de promover una universidad mundialmente, sería a través de la participación en clasificaciones internacionales, como el Ranking Mundial de Universidades del Times Higher Education (THE) y el Ranking Académico de Universidades del Mundo (ARWU - Ranking de Shanghái). La participación en eses rankings muestra que las universidades ansían por promocionarse, atrayendo a más estudiantes extranjeros para obtener más fondos. Sin embargo, es importante tener en cuenta la advertencia presentada por Finardi y Guimarães (2017) y Leite y Genro (2012) en relación a los rankings. Según estos autores, los criterios utilizados para los rankings tienden a favorecer a las universidades del hemisferio norte y/o de habla inglesa y, como tales, eses rankings no pueden captar la realidad (y las especificidades) de las universidades del sur global ${ }^{2}$ (SANTOS, 2011). Como consecuencia de esto, estas clasificaciones no benefician a todos y tampoco benefician a todos los involucrados y partes interesadas de la misma manera (VAVRUS; PEKOL, 2015).

En este contexto, es necesario discutir los rankings como reflejo de un sistema neoliberal globalizante, haciendo que las instituciones de enseñanza superior (IES) se involucren en una rivalidad por recursos cada vez más escasos para el área de la educación. Minto (2019) indica el inicio de una transición a un nuevo modelo de universidad, marcado por la debilidad de la indiferencia entre enseñanza, investigación y extensión y también por la privatización como política de Estado. De esa forma, las instituciones demuestran tener sus actividades cada vez más vinculadas a los objetivos de grandes corporaciones transnacionales. Con el incentivo de la expansión de matrículas en las IES, surge también un sector empresarial con intereses en el potencial lucrativo de la enseñanza superior.

Según Minto (2019), con la posibilidad de alcanzar prestigio y ganancias financieras con el desarrollo de investigaciones científicas, las IES acabaron por realizar investigaciones más alejadas de los intereses populares para aproximarse más a los intereses de las clases dominantes. Así, impulsada por la ola de privatización de los años 1990, la enseñanza superior sufrió los efectos de la reestructuración capitalista global y de la reforma del Estado.

Mientras en los países hegemónicos la producción científica y tecnológica (de los campus universitarios, por ejemplo) une instituciones de punta y sectores productivos, en los países periféricos esa producción acaba no estableciendo relaciones sistemáticas con el sector productivo. Así, el aparato científico y tecnológico de las IES públicas (donde las investigaciones son desarrolladas) pasa a ser visto como "ineficiente", "caro" o incluso "innecesario", generando aún más recortes en las inversiones en educación pública.

\footnotetext{
${ }^{2}$ Según Santos (2011), el sur y el norte globales no son divisiones geográficas - son divisiones epistemológicas, que se utilizan para clasificar los países del norte global como "países centrales" responsables por la producción de ideologías y conocimientos. Los países del sur global son considerados "periféricos" porque tienden a consumir el conocimiento producido en el norte global.
} 
Por consiguiente, las IES públicas con capacidad para producir nuevos conocimientos, ven sus actividades mermadas debido a la escasa inversión realizada en ellas, teniendo que ceder paso a la priorización de sectores de investigación ligados al capital monopolista/imperialista. De esa manera, abriendo camino hacia una mayor mercantilización de la enseñanza superior, en el cual grupos empresariales de diferentes tipos disputan los espacios en un "mercado educativo" en amplia expansión. Productivismo académico, privatizaciones y lógica competitiva/individualista pasan entonces a dominar el escenario de la enseñanza superior.

Para intentar lidiar con este nuevo escenario, urge buscar la construcción/reformulación de instituciones a favor de propósitos sociales, culturales y económicos - un proyecto colectivo de transformación que involucre a toda la sociedad, de la cual las IES son parte integrante y fundamental (MINTO, 2019). En ese sentido, este trabajo tiene como objetivo pensar en un modelo de internacionalización más crítico y sustentable que regionalice y considere el uso de lenguas y las relaciones de poder subyacentes en ese proceso en un contexto particular, el brasileño. Con ese objetivo, partimos de un meta-análisis de investigaciones realizadas en ese contexto para proponer cuestiones de esa reflexión.

\section{Meta-análisis}

Para apoyar la reflexión sobre las posibilidades de una internacionalización más crítica, realizamos un meta-análisis de los estudios realizados por el grupo de investigación al que pertenecen los autores, estudios publicados en los últimos 5 años y en el contexto brasileño, encontrando un total de 26 textos (artículos y capítulos de libros). Los textos incluidos en el meta-análisis fueron 1) Finardi, Amorim y Kawachi, 2018, 2) Finardi y Archanjo (2018), 3) Guimarães y Finardi, 2018, 4) Hildeblando y Finardi, 2018, 5) Vieira, Finardi y Piccin, 2018, 6) Finardi (2017a), 7) Amorim y Finardi (2017), 8) Kawachi, Amorim y Finardi (2017), 9) Finardi y Guimarães (2017), 10) Taquini, Finardi y Amorim (2017), 11) Finardi y França (2016), 12) Finardi, Leão y Pinheiro (2016), 13) Finardi, Santos y Guimarães (2016), 14) Finardi y Ortiz (2015), 15) Finardi y Porcino (2015), 16) Finardi y Prebianca, (2014), 17) Finardi (2014), 18) Finardi y Porcino (2014), 19) Finardi (2016a), 20) Finardi (2016b), 21) Finardi (2017b), 22) Finardi y Tyler (2015), 23) Ortiz y Finardi (2015), 24) Finardi y Archanjo (2015), 25) Porcino y Finardi (2014) y 26) Finardi, Prebianca, Schmitt y Andrade (2014). Fue producido un cuadro con un resumen de los objetivos y los principales hallazgos de estos estudios.

Cuadro 2. Meta-análisis

\begin{tabular}{|l|l|l|l|}
\hline $\mathbf{n}^{\circ}$ & \multicolumn{1}{|c|}{ Texto } & \multicolumn{1}{|c|}{ Objetivo(s) } & \multicolumn{1}{c|}{ Hallazgo(s) Principal(es) } \\
\hline 1 & Finardi, & $\begin{array}{l}\text { Comparar índices de } \\
\text { competencia en inglés e índices } \\
\text { Amorim y } \\
\text { Kawachi, 2018 }\end{array}$ & $\begin{array}{l}\text { Hay una correlación positiva entre índices de } \\
\text { competencia en inglés e índices de } \\
\text { internacionalización }\end{array}$ \\
\hline 2 & $\begin{array}{l}\text { Finardi y } \\
\text { Archanjo, }\end{array}$ & $\begin{array}{l}\text { Analizar el impacto de } \\
\text { programas nacionales como el }\end{array}$ & $\begin{array}{l}\text { El programa CsF incluyó la internacionalización en } \\
\text { la pauta de las universidades brasileñas y tuvo como }\end{array}$ \\
\hline
\end{tabular}




\begin{tabular}{|c|c|c|c|}
\hline & 2018 & $\begin{array}{l}\text { Ciencia sin Fronteras }(\mathrm{CsF}) \text { y el } \\
\text { Idiomas sin Fronteras }(\mathrm{IsF})^{3} \text { en el } \\
\text { proceso de internacionalización } \\
\text { en Brasil }\end{array}$ & $\begin{array}{l}\text { efecto la creación del programa IsF que como } \\
\text { consecuencia, motivó la creación de políticas } \\
\text { lingüísticas en las instituciones de enseñanza } \\
\text { superior brasileñas. }\end{array}$ \\
\hline 3 & $\begin{array}{l}\text { Guimarães y } \\
\text { Finardi, } 2018\end{array}$ & $\begin{array}{l}\text { Revisar los conceptos de } \\
\text { interculturalidad } \\
\text { internacionalización de la } \\
\text { enseñanza superior, discutiendo } \\
\text { la relación existente entre ellos y } \\
\text { el uso de lenguas extranjeras }\end{array}$ & $\begin{array}{l}\text { La interculturalidad, la internacionalización y el } \\
\text { abordaje de intercomprensión se relacionan en la } \\
\text { medida en que tanto la interculturalidad como la } \\
\text { intercomprensión pueden servir para mitigar efectos } \\
\text { negativos de la internacionalización, como el } \\
\text { fortalecimiento de la hegemonía del inglés como } \\
\text { lengua franca académica. }\end{array}$ \\
\hline 4 & $\begin{array}{l}\text { Hildeblan } \\
\text { Finardi, } 2\end{array}$ & $\begin{array}{l}\text { Analizar las posibilidades } y \\
\text { limitaciones del enfoque de } \\
\text { aprendizaje internacional (COIL) } \\
\text { para fomentar la colaboración } \\
\text { internacional } y \text { y la } \\
\text { internacionalización. }\end{array}$ & $\begin{array}{l}\text { COIL es un enfoque que puede promover la } \\
\text { movilidad académica en línea y la } \\
\text { internacionalización, aunque la colaboración puede } \\
\text { mejorar la competencia intercultural cuando se } \\
\text { combina con el enfoque de intercomprensión para } \\
\text { permitir el uso de diferentes idiomas. }\end{array}$ \\
\hline 5 & y Piccin, & $\begin{array}{l}\text { Analizar los desafíos a los que se } \\
\text { enfrentan los Institutos Federales } \\
\text { de Educación, Ciencia y } \\
\text { Tecnología (IFs) para } \\
\text { internacionalizarse. }\end{array}$ & $\begin{array}{l}\text { Los resultados sugieren que las lenguas extranjeras, } \\
\text { en general, y el inglés, en particular, desempeñan un } \\
\text { papel crucial en la internacionalización de las } \\
\text { universidades federales en Brasil. El análisis } \\
\text { cualitativo de los datos de los institutos federales } \\
\text { sugiere que el escenario en ese contexto, no es muy } \\
\text { diferente y que el inglés también tiene un papel } \\
\text { importante en la superación de desafíos para que los } \\
\text { institutos federales se internacionalicen. Además, los } \\
\text { datos de los dos institutos federales sugieren que la } \\
\text { internacionalización en ese contexto sigue siendo } \\
\text { pasiva y dependiente de programas nacionales tales } \\
\text { como el CsF y el IsF y predominantemente } \\
\text { enfocados en la movilidad académica para el } \\
\text { exterior. }\end{array}$ \\
\hline 6 & $\begin{array}{l}\text { Finard1 } \\
(2017 a)\end{array}$ & $\begin{array}{l}\text { Comparar las políticas } \\
\text { lingüísticas de Brasil y Suiza } \\
\text { para proponer el uso del enfoque } \\
\text { de intercomprensión para la } \\
\text { enseñanza de idiomas extranjeros } \\
\text { en Brasil }\end{array}$ & $\begin{array}{l}\text { Brasil es un país multilingüe de hecho donde el } \\
\text { inglés es la principal lengua extranjera en la } \\
\text { enseñanza básica y superior. Es necesario pensar } \\
\text { enfoques más multilingües para incluir otros idiomas } \\
\text { en la enseñanza brasileña. }\end{array}$ \\
\hline 7 & $\begin{array}{l}\text { Amorim \& } \\
\text { Finardi (2017) }\end{array}$ & $\begin{array}{l}\text { Estudio de caso para analizar la } \\
\text { internacionalización en tres } \\
\text { niveles: macro (acciones } \\
\text { gubernamentales), meso } \\
\text { (institución) y micro (comunidad } \\
\text { académica). }\end{array}$ & $\begin{array}{l}\text { Macro (las acciones más importantes son los } \\
\text { programas Ciencias sin Fronteras (CsF) e Idiomas } \\
\text { sin Fronteras (IsF), meso (los académicos están } \\
\text { dispuestos pero no totalmente comprometidos con la } \\
\text { internacionalización) y micro (bajo nivel de } \\
\text { competencia en lenguas extranjeras afecta la } \\
\text { internacionalización). }\end{array}$ \\
\hline 8 & $\begin{array}{l}\text { Kawachi; } \\
\text { Amorim \& } \\
\text { Finardi (2017) }\end{array}$ & $\begin{array}{l}\text { Estudio de caso para analizar el } \\
\text { interfaz entre TOEFL ITP, } \\
\text { internacionalización y evaluación } \\
\text { de idiomas. }\end{array}$ & $\begin{array}{l}\text { Las puntuaciones del TOEFL ITP se correlacionan } \\
\text { con las acciones y programas de } \\
\text { internacionalización. }\end{array}$ \\
\hline 9 & Finardi \& & Analizar la situación brasileña en & icas lingüísticas están asociadas con la \\
\hline
\end{tabular}

\footnotetext{
${ }^{3}$ IsF (Idiomas sin Fronteras) fue un programa financiado por el gobierno brasileño hasta el primer semestre del 2019 para promover el dominio de idiomas en la educación superior brasileña con la oferta de clases presenciales, cursos en línea y exámenes de los siguientes idiomas: inglés, francés, alemán, italiano, español, japonés y portugués como lengua extranjera.
} 


\begin{tabular}{|c|c|c|c|}
\hline & $\begin{array}{l}\text { Guimarães } \\
\text { (2017) }\end{array}$ & $\begin{array}{l}\text { términos de rankings y políticas } \\
\text { lingüísticas para la } \\
\text { internacionalización. }\end{array}$ & $\begin{array}{l}\text { internacionalización, pero las clasificaciones no } \\
\text { captan la realidad en Brasil }\end{array}$ \\
\hline 10 & $\begin{array}{l}\text { Taquini; } \\
\text { Finardi \& } \\
\text { Amorim } \\
(2017)\end{array}$ & $\begin{array}{l}\text { Análisis de la oferta de cursos en } \\
\text { inglés (EMI) en Turquía y } \\
\text { comparación con la oferta en } \\
\text { Brasil. }\end{array}$ & $\begin{array}{l}\text { Similitud entre Turquía y Brasil: ambos quieren } \\
\text { ofrecer cursos de EMI para internacionalizarse. } \\
\text { Diferencia: en Turquía, el EMI se ofrece } \\
\text { principalmente en instituciones privadas, mientras } \\
\text { que en Brasil lo es en instituciones públicas. }\end{array}$ \\
\hline 11 & $\begin{array}{l}\text { Finardi \& } \\
\text { França (2016) }\end{array}$ & $\begin{array}{l}\text { Analizar el papel del inglés en la } \\
\text { producción académica brasileña } \\
\text { del área de lingüística. }\end{array}$ & $\begin{array}{l}\text { La circulación e impacto de la producción académica } \\
\text { brasileña se ve afectada negativamente por la falta de } \\
\text { publicaciones en inglés. }\end{array}$ \\
\hline 12 & $\begin{array}{l}\text { Finardi; Leão } \\
\text { \& Pinheiro } \\
(2016)\end{array}$ & $\begin{array}{l}\text { Revisar las políticas lingüísticas } \\
\text { y el uso del enfoque de } \\
\text { enseñanza de contenidos por } \\
\text { medio de la lengua (CLIL) para } \\
\text { la internacionalización en Brasil. }\end{array}$ & $\begin{array}{l}\text { El enfoque CLIL no es muy utilizado en Brasil } \\
\text { principalmente debido a la falta de políticas } \\
\text { lingüísticas y de formación docente para el uso de } \\
\text { ese tipo de abordaje. }\end{array}$ \\
\hline 13 & $\begin{array}{l}\text { Finardi; Santos } \\
\text { \& Guimarães } \\
\text { (2016) }\end{array}$ & $\begin{array}{l}\text { Describir la creación de una } \\
\text { división de idiomas en la oficina } \\
\text { internacional de una universidad } \\
\text { brasileña. }\end{array}$ & $\begin{array}{l}\text { Existe una fuerte interfaz entre el lenguaje y las } \\
\text { acciones y políticas de internacionalización. }\end{array}$ \\
\hline 14 & $\begin{array}{l}\text { Finardi \& } \\
\text { Ortiz (2015) }\end{array}$ & $\begin{array}{l}\text { Analizar las motivaciones para } \\
\text { internacionalizar una universidad } \\
\text { pública y privada brasileña. }\end{array}$ & $\begin{array}{l}\text { Las universidades públicas brasileñas están } \\
\text { motivadas (con fines académicos) a } \\
\text { internacionalizarse mientras que las universidades } \\
\text { privadas no lo están (debido al tamaño del mercado } \\
\text { interno) }\end{array}$ \\
\hline 15 & $\begin{array}{l}\text { Finardi \& } \\
\text { Porcino (2015) }\end{array}$ & $\begin{array}{l}\text { Analizar el uso del inglés en la } \\
\text { internacionalización y educación } \\
\text { brasileña. }\end{array}$ & $\begin{array}{l}\text { El inglés tiene un papel funcional en la } \\
\text { internacionalización y un papel formativo en la } \\
\text { educación. }\end{array}$ \\
\hline 16 & $\begin{array}{l}\text { Finardi \& } \\
\text { Prebianca, } \\
(2014)\end{array}$ & $\begin{array}{l}\text { Estudio de caso para analizar el } \\
\text { papel de las políticas lingüísticas, } \\
\text { la internacionalización y las } \\
\text { tecnologías en un curso de } \\
\text { formación de profesores de } \\
\text { inglés. }\end{array}$ & $\begin{array}{l}\text { El currículo del curso de grado de enseñanza } \\
\text { analizado no garantiza la enseñanza de aspectos } \\
\text { importantes relacionados con esos tres temas. }\end{array}$ \\
\hline 17 & Finardi (2014) & $\begin{array}{l}\text { Proponer la apropiación del } \\
\text { inglés como idioma internacional } \\
\text { en Brasil. }\end{array}$ & $\begin{array}{l}\text { Las políticas lingüísticas pueden crear una brecha } \\
\text { social, ya que solo aquellos que puedan aprender } \\
\text { inglés en cursos privados lo harán en Brasil. }\end{array}$ \\
\hline 18 & $\begin{array}{l}\text { Finardi \& } \\
\text { Porcino (2014) }\end{array}$ & $\begin{array}{l}\text { Reflexionar sobre la interfaz } \\
\text { entre tecnología, metodología, } \\
\text { internacionalización y } \\
\text { globalización. }\end{array}$ & $\begin{array}{l}\text { Estos cuatro temas están muy asociados en la } \\
\text { enseñanza del idioma inglés. }\end{array}$ \\
\hline 19 & $\begin{array}{l}\text { Finardi } \\
(2016 a)\end{array}$ & $\begin{array}{l}\text { Revisar las políticas lingüísticas } \\
\text { en Brasil. }\end{array}$ & $\begin{array}{l}\text { El inglés tiene el papel de una lengua extranjera en la } \\
\text { educación básica y de una lengua internacional en la } \\
\text { enseñanza superior. }\end{array}$ \\
\hline 20 & $\begin{array}{l}\text { Finardi } \\
(2016 b)\end{array}$ & $\begin{array}{l}\text { Describir el uso del inglés como } \\
\text { idioma global en Brasil desde } \\
\text { una perspectiva local. }\end{array}$ & $\begin{array}{l}\text { El inglés amenaza otros idiomas adicionales } \\
\text { (extranjeros, de herencia e indígenas) en Brasil. }\end{array}$ \\
\hline 21 & $\begin{array}{l}\text { Finardi } \\
(2017 b)\end{array}$ & $\begin{array}{l}\text { Analizar la interfaz entre } \\
\text { globalización e inglés en Brasil. }\end{array}$ & $\begin{array}{l}\text { La globalización ha impactado el uso del inglés en } \\
\text { Brasil }\end{array}$ \\
\hline 22 & $\begin{array}{l}\text { Finardi \& } \\
\text { Tyler (2015) }\end{array}$ & $\begin{array}{l}\text { Proponer una matriz para la } \\
\text { adaptación de los MOOC para } \\
\text { personas que no hablan inglés. }\end{array}$ & $\begin{array}{l}\text { El inglés es muy importante en el acceso a la } \\
\text { educación en línea. }\end{array}$ \\
\hline 23 & $\begin{array}{l}\text { Ortiz \& } \\
\text { Finardi (2015) }\end{array}$ & $\begin{array}{l}\text { Describir un proyecto utilizando } \\
\text { CLIL para incluir socialmente a } \\
\text { inmigrantes y refugiados. }\end{array}$ & $\begin{array}{l}\text { La enseñanza de contenidos y lenguaje juntos puede } \\
\text { fomentar el desarrollo de la inclusión social. }\end{array}$ \\
\hline 24 & $\begin{array}{l}\text { Finardi \& } \\
\text { Archanjo } \\
(2015)\end{array}$ & $\begin{array}{l}\text { Analizar las políticas lingüísticas } \\
\text { en Brasil. }\end{array}$ & $\begin{array}{l}\text { Las políticas lingüísticas no son consistentes y } \\
\text { convergentes con los objetivos de } \\
\text { internacionalización. }\end{array}$ \\
\hline 25 & Porcino \& & Analizar la interfaz entre la & ELT está altamente asociado a la \\
\hline \multicolumn{3}{|c|}{ (C) Rev. Inter. Educ. Sup. } & $\mathrm{e} 020031$ \\
\hline
\end{tabular}




\begin{tabular}{|c|c|c|c|}
\hline & Finardi (2014) & $\begin{array}{l}\text { internacionalización y la } \\
\text { enseñanza del idioma inglés } \\
\text { (ELT) en Brasil. }\end{array}$ & internacionalización. \\
\hline 26 & $\begin{array}{l}\text { Finardi; } \\
\text { Prebianca; } \\
\text { Schmitt \& } \\
\text { Andrade } \\
\text { (2014) }\end{array}$ & $\begin{array}{l}\text { Reflexionar sobre la interfaz } \\
\text { entre tecnología, metodología e } \\
\text { internacionalización a través del } \\
\text { análisis del curso My English } \\
\text { Online (MEO) ofertado por el } \\
\text { programa IsF. }\end{array}$ & $\begin{array}{l}\text { El curso MEO no es suficiente para garantizar el } \\
\text { dominio del inglés y tampoco las mejoras en el } \\
\text { aprendizaje y la internacionalización. }\end{array}$ \\
\hline
\end{tabular}

Fuente: Los autores.

El meta-análisis mostró que las lenguas adicionales tienen un papel crucial en el proceso de internacionalización de la enseñanza superior en Brasil. Además, los resultados del meta-análisis sugieren que el proceso de internacionalización brasileña sigue siendo pasivo (ejemplo LIMA; MARANHÃO, 2009), reactivo y dependiente de iniciativas gubernamentales de programas como el CsF, el IsF y la financiación a través de ediciones como el Programa Institucional de Internacionalización (PrInt) de la agencia del gobierno de Coordinación de Perfeccionamiento del Personal del Nivel Superior (Capes). Sin embargo, el hallazgo más importante del meta-análisis es que existe una brecha entre los principios y las políticas lingüísticas para guiar el proceso de internacionalización en Brasil. Sobre la base de este hallazgo, se proponen principios para pensar en una internacionalización más crítica, sustentable y activa que pueda regionalizar o glocalizar (PATEL; LYNCH, 2013) el proceso de internacionalización de la enseñanza superior en Brasil.

\section{Metodología}

La internacionalización es un fenómeno bastante reciente en Brasil (en comparación con Europa y EE. UU., por ejemplo), por lo que los grupos de investigación emergentes están abordando este tema en los últimos años. El grupo cuyas publicaciones fueron elegidas para el meta-análisis ${ }^{4}$ de este artículo empezó un proyecto de investigación sobre la internacionalización de la enseñanza superior en Brasil en el 2012 y después de más de media década de investigaciones y producciones, entendió que era necesario hacer una pausa para analizar los resultados de esas investigaciones de forma sistemática. Para ello, el grupo de investigaciones seleccionó una cantidad relevante de publicaciones en esta área para el metaanálisis. La novedad y contribución de este artículo es doble: resumir los hallazgos de esos estudios y analizar los resultados de dichos estudios presentando una visión general de la internacionalización en Brasil, comparando con la investigación actual en este tema.

Un hallazgo importante del meta-análisis sugirió una brecha (y desajuste) de principios y políticas (especialmente en términos del uso de idiomas) para guiar el proceso de

\footnotetext{
${ }^{4}$ El meta-análisis ha sido una metodología muy relevante para la investigación en los últimos años (en el ámbito de las ciencias de la educación, por ejemplo), según lo descrito por Sánchez-Meca y Botella (2010) y SánchezMeca (2010). Es un tipo de investigación científica que tiene como propósito integrar de forma objetiva y sistemática los resultados de los estudios sobre un determinado problema de investigación.
} 
internacionalización y su evaluación en Brasil. Sobre la base de este hallazgo, se propone un marco tentativo para abordar la internacionalización de forma más crítica.

\section{Políticas Lingüísticas}

Finardi (2016a, 2016b, 2017b), en un análisis del escenario lingüístico en Brasil se concluye que, aunque hay muchos idiomas que se hablan en ese país (de hecho, más de 200), Brasil es principalmente un país monolingüe en el que el idioma nacional (portugués) excluyó otros idiomas nativos (en su mayoría, idiomas indígenas y de herencia) y donde el inglés también excluyó otros idiomas extranjeros, esto como consecuencia de las últimas reformas educativas.

Las últimas reformas educativas incluyen el uso obligatorio del inglés como idioma extranjero que se enseñará en la educación primaria (en la versión anterior de la Ley de Directrices para la Educación [LDB, en portugués], las escuelas podían elegir el idioma extranjero que se enseñaría, de acuerdo con las necesidades locales - Ley No. 13.415/2017) ${ }^{5}$. Esto tuvo consecuencias negativas para la enseñanza de otros idiomas como el español, el francés y el italiano, que ya no son "necesarios" en la educación primaria. Otras reformas incluyen la Base Nacional Curricular Común (BNCC) ${ }^{6}$, que cambió el sistema educativo en todo Brasil, incluidos los temas relacionados con la enseñanza, el aprendizaje y el uso de idiomas.

Las discusiones sobre idiomas y políticas lingüísticas parecen crear una brecha entre los lingüistas y la opinión pública. Rajagopalan (2003, p.129) afirma que los lingüistas tienden a centrarse en los efectos negativos de la globalización (y sus consecuencias) y tratan de crear medidas de protección para preservar determinados idiomas. El sentido común ha sido visto como un obstáculo para eso y los expertos en idiomas tienden a mantenerse alejados de la gente común, una forma de elitismo intelectual y desprecio por las opiniones públicas. De esta manera, se crea una brecha entre el mundo académico y la sociedad.

Según Santos (2009), el sentido común no resulta de una práctica diseñada intencionalmente para este propósito, ya que este se reproduce espontáneamente en la vida cotidiana. El autor también afirma que el sentido común "desconfía de la opacidad de los objetivos tecnológicos y el esoterismo del conocimiento para favorecer el principio de igualdad de acceso al discurso, la competencia cognitiva y la competencia lingüística" (SANTOS, 2009, p. 108, nuestra traducción). Cuando la discusión es sobre idiomas, las preocupaciones de todas las personas deberían ser consideradas, no importando si son expertos o laicos en el tema, esto porque todos usamos los idiomas para diversos propósitos. Además, trabajar con idiomas significa intervenir en las realidades sociales que crean los lenguajes (RAJAGOPALAN, 2003, p.126).

\footnotetext{
${ }^{5}$ Más información en: http://www.planalto.gov.br/ccivil_03/_Ato2015-2018/2017/Lei/L13415.htm

${ }^{6}$ Más información en: http://basenacionalcomum.mec.gov.br/

\begin{tabular}{l|c|c|c|c|c}
\hline () Rev. Inter. Educ. Sup. & Campinas, SP & v.6 & $1-23$ & $\mathrm{e} 020031$ & 2020 \\
\hline
\end{tabular}
}


Al definir las necesidades locales de las instituciones de enseñanza superior, podemos tratar de encontrar un equilibrio entre las fuerzas globales y locales. Siguiendo a Finardi y Csillagh, (2016) y Guimarães y Finardi (en prensa), sugerimos que las políticas lingüísticas institucionales se analicen en seis dimensiones de la enseñanza superior, a saber, el: 1) idioma de admisión; 2) enseñanza de idiomas; 3 ) idioma de instrucción; 4) idioma de investigación; 5) idioma de administración y 6) idioma de comunicación externa.

El idioma (o idiomas) de admisión (1) es el que utiliza la institución para seleccionar estudiantes, generalmente a través de exámenes de ingreso. La enseñanza de idiomas (2) debe tener en cuenta los idiomas que se consideran valiosos para que las universidades y los estudiantes logren sus objetivos, especialmente los relacionados con la internacionalización. El idioma de instrucción (3) es el idioma (o idiomas) que se utiliza para educar a los estudiantes, dependiendo de su campo de estudio. El idioma en el punto 4 se refiere de forma específica al idioma de cooperaciones internacionales para investigación, el 5 al idioma de las comunicaciones institucionales internas y el 6 al idioma de las comunicaciones con otras instituciones.

\section{Internacionalización Crítica}

Ante tantos riesgos y posibilidades ligadas al tema de internacionalización, una visión crítica sobre esa temática debe ser considerada. Lima y Maranhão (2009) tratan del concepto de internacionalización activa y pasiva que puede ser analizado desde una perspectiva crítica. Reducida a pocos países, la internacionalización "activa" está ligada a una internacionalización hegemónica derivada de una globalización también hegemónica, en la que unos pocos países cosechan los beneficios de la globalización, mientras que muchos países sufren las consecuencias negativas de esa misma globalización. Así, se amplían los desequilibrios entre las regiones más y menos beneficiadas, manifestándose un "flagrante descompaso entre los activos conquistados en los países centrales y periféricos" (LIMA; MARANHÃO, 2009, p. 583, nuestra traducción).

En ese sentido, Leite y Genro (2012) creen que la internacionalización trae una nueva epistemología de la enseñanza superior, una que defiende la homogeneización de los currículos y del sistema de evaluación de las instituciones. Según los autores, las instituciones de enseñanza superior alrededor del mundo son evaluadas por medio de indicadores de calidad establecidos por el modelo institucional de los países hegemónicos. Con eso en mente, las instituciones de los países periféricos buscan la estandarización de sus currículos académicos para adecuarse al modelo hegemónico y favorecer su resultado en la evaluación por los indicadores de calidad globales (también desarrollados en el norte global). Con una buena evaluación, y luego bien posicionada en el ranking internacional, la institución gana credibilidad y más posibilidades de recibir inversiones. Sin embargo, al priorizar su calificación global, las instituciones ignoran la realidad de su contexto y las prácticas locales. 
De esta forma, las autoras (LEITE; GENRO, 2012) entienden la evaluación y clasificación global de las instituciones como siendo una estrategia de mercantilización de la enseñanza superior. Las instituciones de los países periféricos son convencidas a recorrer los caminos definidos por modelos hegemónicos en sus prácticas y políticas. En este contexto, valores como competencia y búsqueda por la rentabilidad hacen instituciones educativas similares a las empresas y con ello, la formación social y política de los sujetos va dando lugar a una formación regida por la productividad y la eficiencia.

A esta reproducción de modelos (principalmente educacionales), Lima y Maranhão (2009) llaman de internacionalización "pasiva", la que es definida en los países centrales y aplicada a los países periféricos (sin las debidas adaptaciones a las necesidades locales). Las consecuencias de ese tipo de internacionalización pasiva son desastrosas, entre las cuáles podemos mencionar la mercantilización de la enseñanza superior y la presencia de empresas extranjeras interesadas en explotar al máximo el potencial lucrativo de la enseñanza superior local. Las señales de una internacionalización pasiva incluyen también un desequilibrio en la movilidad académica global, es decir, una cantidad mucho mayor de alumnos oriundos de países periféricos buscando estudios en países centrales, de que el camino opuesto (alumnos de países centrales que van a estudiar en países periféricos).

Se entiende que esa desigualdad es debido a las diferentes motivaciones que llevan a los países del norte y del sur a trabajar por la internacionalización. Morosini y Corte (2018) analizaron los estudios de maestría y doctorado (acerca de la internacionalización) en Brasil en los últimos cinco años y afirman que en ellos la movilidad es vista como la principal estrategia de internacionalización. Las autoras concluyeron que mientras los países del norte han trabajado por una internacionalización activa con motivaciones económicas, estos países han asumido también, el rol de centralizadores del conocimiento. Estos países buscan así, atraer estudiantes promisores de los países del sur, que (por su lado) son motivados mayoritariamente por la integración regional y entre continentes. Por eso, intentan desarrollar su comunidad académica y disminuir las desigualdades entre las naciones. Sin embargo, aún se percibe que los países del Sur, entre ellos Brasil, siguen buscando los nuevos conocimientos principalmente en los países hegemónicos, desvalorizando los conocimientos locales y cediendo a la transnacionalización de la enseñanza superior por medio de su mercantilización. De hecho, ese es uno de los hallazgos de Finardi y Archanjo (2018) y de Vieira, Finardi y Piccin (2018).

De forma similar, Maués y Souza (2018) entienden que la internacionalización de la enseñanza superior, con foco en la movilidad con otras motivaciones que no sean la búsqueda por interculturalidad e integración, se puede denominar transnacionalización. Para ellos, las políticas de internacionalización en Brasil, han reforzado esa mercantilización neoliberal de la enseñanza superior, por medio de la transnacionalización. Estos autores mencionan el programa CsF, programa que tenía como principal objetivo dar una formación de calidad en instituciones extranjeras y atraer a Brasil estudiantes e investigadores del extranjero que pudieran contribuir para el desarrollo del país. Ese programa tuvo el apoyo de diversas

\begin{tabular}{c|c|c|c|c|c|c} 
empresas privadas que buscaban & absorber & esos & futuros profesionales, bien e \\
\hline () Rev. Inter. Educ. Sup. & Campinas, SP & v.6 & $1-23$ & e020031 & 2020 \\
\hline
\end{tabular}


internacionalmente calificados en sus equipos. Por consiguiente, es posible concluir que no solamente la movilidad en el ámbito del CsF tuvo motivaciones fuertemente económicas, sino que también, reforzaban una desvalorización de la educación nacional y regional al buscar la formación de calidad en el extranjero, principalmente en los países hegemónicos. Finardi y Archanjo (2018) corroboran esa interpretación, añadiendo que el programa CsF tuvo también un importante (y no siempre positivo) efecto en las políticas lingüísticas y de internacionalización en Brasil.

Para Andreotti y colegas (2016) ese desequilibrio es justamente fruto, por un lado, de esa asimetría entre los países en el imaginario global, resultante del riesgo que el proceso de internacionalización presenta de reforzar el imaginario jerárquico de dominación intelectual y, por otro lado, cultural de los países del Norte sobre los del Sur. Los autores también afirman que algunas iniciativas educativas para la internacionalización han alimentado esa lógica jerárquica. Los países del Norte son tenidos como desarrollados y productores del conocimiento mientras que los del Sur, son vistos como atrasados y consumidores del conocimiento. Los autores creen que uno de los mayores desafíos de la internacionalización, es el de proponer iniciativas que cuestionen y deconstruyan esa lógica jerárquica, que tiene su origen en el sistema de pensamiento occidental moderno.

Este sistema de pensamiento está formado por distinciones visibles basadas en
distinciones invisibles, consecuencia de una lógica que define y reconoce como
realidad social, todo aquello que se encuentra "en este lado de la línea abisal", o sea,
en el otro lado de la línea abisal. Esta separación radical que percibe sólo uno de los
lados de ese abismo cognitivo como "existente, visible, inteligible, presente" y el
otro necesariamente como "inexistente, invisible, ininteligible, ausente",
estableciendo una absoluta hegemonía cognitiva sin posibilidad alguna de la
copresencia y coexistencia de esos dos lados, es la esencia del pensamiento abisal.
(ANDREOTTI et al., 2016, p. 135-136, nuestra traducción)

Según esos autores, para la deconstrucción de ese imaginario hegemónico, es necesario primordialmente que la educación lleve a los alumnos a percibir la multiplicidad y pluralidad de saberes en el mundo, más allá de la realidad visible y trabajar para una coexistencia pacífica. En esta misma línea de pensamiento, Patel y Lynch (2013) critican los modelos más comunes de internacionalización por enfocarse más en una aculturación por parte de los extranjeros que en un intercambio de conocimientos académicos y culturales. Teniendo en cuenta eso, los autores proponen que la enseñanza superior se oriente hacia la glocalización, o sea, la conexión de los contextos locales y globales, considerando los importantes aportes que diferentes comunidades y contextos culturales pueden traer, sin que una cultura o perspectiva se sobreponga y/o menosprecie a la otra. Esto también fue discutido por Guimarães y Finardi (2018) y revisado en el meta-análisis. Los autores sugieren que las instituciones de la enseñanza superior sean más proactivas y desarrollen estrategias dentro de un currículo de aprendizaje glocalizado, basado en los principios de responsabilidad, justicia y sustentabilidad social. Esto posibilita ir más allá de la tolerancia y acomodación intercultural y acercarse más de acciones que, de hecho, muestren el reconocimiento de la coexistencia de las diferentes culturas. 
Paralelamente, ante la lógica mercadológica y de aculturación que la internacionalización ha asumido, Piccin y Finardi (2019) proponen un énfasis en los contextos locales que se puedan relacionar con la solidaridad entre las instituciones del Sur. Permitiendo que su internacionalización pueda ser descolonizada y traiga beneficios para una democracia más participativa. Las autoras (PICCIN; FINARDI, 2019, p. 15, nuestra traducción) sugieren que para una internacionalización más crítica es

\begin{abstract}
necesario que el Sur localice el Sur, y no sólo el Norte, dejando de reproducir tales relaciones de dependencia sea con los países del Norte o con sus pares del Sur, como en el caso antes mencionado. Es urgente también comprender las complejas relaciones e intereses que rigen la internacionalización de la educación mundial, en la búsqueda de alternativas a los patrones hegemónicos y homogeneizantes, reflexionando críticamente sobre las concepciones de internacionalización que hemos adoptado. Para que esas concepciones no aporten conceptos que puedan beneficiar a algunos en detrimento de otros [...] es necesario desarrollar esos conceptos a través de la reflexión crítica, considerando los contextos y las demandas de las IES, así como las estrategias que puedan orientar un proceso de internacionalización activo, como Lima y Maranhão (2009).
\end{abstract}

Bernheim (2018) cree que la internacionalización puede llegar a ser una respuesta de las instituciones frente a los impactos homogeneizadores de la globalización, de forma a respetar sus individualidades locales. Para tanto es necesario repensar la internacionalización según los contextos locales para actuar globalmente por medio de "una cooperación internacional solidaria con énfasis en la cooperación horizontal, basada en el diálogo intercultural y respetuosa de la idiosincrasia e identidad de los países participantes, así como el diseño de redes interuniversitarias y de espacios académicos ampliados" (BERNHEIM, 2018, p. 261).

Sin embargo, como ha sido destacado por Maués y Souza (2018), la mayor parte de las iniciativas gubernamentales relacionadas con la internacionalización no han tenido un carácter solidario enfocado en mutua cooperación, sino que un carácter más neocolonialista por medio de movimientos verticales y unilaterales. La iniciativa más reciente del gobierno brasileño es el Programa PrInt, de apoyo económico a la internacionalización de la enseñanza superior. Dicha iniciativa fue materializada por medio de su agencia Capes y posee enfoque principalmente en el nivel de postgrado. Ese programa tiene el objetivo de:

Fomentar la construcción, la implementación y la consolidación de planes estratégicos de internacionalización de las instituciones contempladas en las áreas del conocimiento por ellas priorizadas; Estimular la formación de redes de investigaciones internacionales con el objetivo de mejorar la calidad de la producción académica vinculadas al posgrado; Ampliar las acciones de apoyo a la internacionalización en el posgrado de las instituciones contempladas; Promover la movilidad de docentes y discentes, con énfasis en doctorandos, postdoctorandos y docentes para el extranjero y del extranjero para Brasil, vinculados a programas de postgrado stricto sensu con cooperación internacional; Fomentar la transformación de las instituciones participantes en un ambiente internacional; e Integrar otras acciones de fomento de Capes al esfuerzo de internacionalización. (CAPES, 2017, p. 1, nuestra traducción)

Se puede percibir en los objetivos del programa, un incentivo a una internacionalización más activa de las instituciones, esto por medio de la elaboración de 
estrategias de internacionalización para no solamente reaccionar a las propuestas de las universidades extranjeras. Sin embargo, el hecho de que ese programa liste 26 países (Alemania, Argentina, Australia, Austria, Bélgica, Canadá, China, Corea del Sur, Dinamarca, España, Estados Unidos de América, Finlandia, Francia, India, Irlanda, Italia, Japón, México, Noruega, Nueva Zelanda, Países Bajos, Reino Unido, Rusia, Sudáfrica, Suecia, Suiza), la mayoría de los cuales está en el Norte Global y para donde esa movilidad se debe enfocar, es señal de que la internacionalización brasileña, al contrario de lo que proponen Piccin y Finardi (2019), todavía mira hacia al Norte. Además, aunque la movilidad es uno de los objetivos del programa, el programa incita a una expansión de la visión de las estrategias de internacionalización para una que contemple también la formación de redes y la preparación del propio ambiente institucional. Sin embargo, como establecido en el Anexo 1 de la llamada pública del programa (CAPES, 2017), se determina que 70\% de las acciones sean direccionadas para países, en su mayoría considerados hegemónicos, conforme subrayamos arriba, perpetuando la lógica jerárquica dominante, sin intenciones solidarias.

Canzani y Araujo Filho (2018) señalan que la idea de internacionalización solidaria, posible por medio de la integración de las instituciones de enseñanza superior en redes, y cuando orientada a la complementariedad y al fortalecimiento local, puede ser una alternativa para confrontar la mercantilización de la enseñanza superior. Además, la educación podría mantenerse como un bien público, con el objetivo de disminuir la desigualdad entre los países. "La internacionalización converge en la región para la integración de la IES mientras que la integración regional favorece la internacionalización, por tanto "internacionalizar regionalizando' (NETTO, 2016, p. 291), sintetiza adecuadamente esta 'simbiótica' relación.” (CANZANI; ARAUJO FILHO, 2018, p.168).

En la región de Latinoamérica y El Caribe, esa alternativa ha sido encontrada por algunas instituciones de enseñanza superior por medio de la construcción del Espacio de Encuentro Latinoamericano y Caribeño de Educación Superior (ENLACES). Ese espacio fue creado a partir de una propuesta de la Conferencia Regional de Educación Superior en Latinoamérica (CRES) de 2018, que tiene como objetivo integrar las instituciones y fortalecer el sistema universitario regional, para así confrontar la mercantilización de la educación y disminuir la brecha entre sus países y los países vistos como desarrollados (GUARGA; SOSA, 2018).

Así, se entiende que, para promover una internacionalización más crítica en Brasil, es necesario rever las motivaciones detrás de las políticas gubernamentales de internacionalización. Por lo tanto, uno podría pensar pensar en propuestas más glocales y relaciones más solidarias entre las instituciones. Por ahora, es posible que las instituciones y sus agentes trabajen en las brechas de las políticas gubernamentales, para orientar su comunidad académica a acciones locales y globales más responsables y sustentables socialmente. 


\section{Conclusión}

Este trabajo tuvo como objetivo reflexionar sobre la internacionalización de la enseñanza superior de forma más crítica a partir de un meta-análisis de los estudios realizados por el grupo de investigación al que pertenecen los autores de este artículo. El análisis de dichos estudios trae algunas consideraciones importantes con respecto a la interfaz entre las políticas lingüísticas y la internacionalización. Los resultados generales del meta-análisis sugieren que existe una brecha entre los principios y las políticas (lingüísticas) para guiar a las instituciones brasileñas de enseñanza superior en sus procesos de internacionalización. Para superar esta brecha, los autores proponen que las instituciones brasileñas de enseñanza superior reflexionen y planifiquen sus acciones considerando las políticas lingüísticas y la internacionalización crítica.

La primera dimensión muestra que los idiomas tienen un papel clave para la internacionalización de las instituciones de enseñanza superior. Por lo tanto, para hacer un uso efectivo de los idiomas, sugerimos que las universidades creen políticas lingüísticas para la internacionalización, teniendo en cuenta sus propias necesidades, considerando seis aspectos relacionados con el uso de idiomas en el escenario de enseñanza superior, a saber: el idioma de admisión; enseñanza de idiomas; el idioma de instrucción; el idioma de la investigación; el idioma de la administración; y el idioma de la comunicación externa.

La segunda dimensión trae los aspectos colonialistas que la internacionalización puede asumir por medio de la mercantilización de la enseñanza superior y del aumento de la brecha entre los países hegemónicos y periféricos. Con base en eso, el gobierno y las instituciones de enseñanza superior deben reflexionar sobre sus motivaciones para la internacionalización y buscar una internacionalización solidaria por medio de la integración y colaboración regional para pensar localmente y a partir de eso actuar globalmente de manera de que pueda ser disminuida la desigualdad y des-construir la lógica jerárquica dominante.

Finalmente, es importante destacar el papel de las redes de colaboración, como ENLACES. A través de estas redes, los países e instituciones que comparten desafíos similares pueden trabajar juntos para confrontar los valores hegemónicos de la globalización y de la internacionalización pasiva y acrítica. Esto es porque tales redes son elementos importantes para la integración regional y para la internacionalización solidaria. Además, dichas redes tienen el potencial de contra-restar fuerzas hegemónicas de la internacionalización en el Norte Global por medio de la integración y articulación regional con acuerdos más equilibrados y bilaterales para las universidades involucradas.

\section{Referencias}

ALTBACH, Philip G.; KNIGHT, Jane. Visión panorámica de la internacionalización en la educación superior: motivaciones y realidades. Perfiles educativos, v. 28, n. 112, p. 13-39, 2006. 
AMORIM, Gabriel Brito; FINARDI, Kyria Rebeca. Globalização e internacionalização do ensino superior: Evidências de um estudo de caso nos níveis micro, meso e macro.

Avaliação: Revista da Avaliação da Educação Superior, 2017.

ANDREOTTI, Vanessa Oliveira; PEREIRA, Renato.; EDMUNDO, Eliana; ARAÚJO, F. Internacionalização da educação brasileira: possibilidades, paradoxos e desafios. In: LUNA, José Marcelo Freitas (org.). Internacionalização do currículo: educação, interculturalidade, cidadania global. Campinas: Pontes, 2016, p. 129-154.

BEELEN, Jos; JONES, Elspeth. Redefining internationalization at home. In: CURAJ, Adrian; MATEI, Liviu; PRICOPIE, Remus; SALMI, Jamil; SCOTT, Peter (Org.). The European Higher Education Area, 2015.

BERNHEIM, Carlos Tunnermann. Los desafíos de la universidad en el Siglo XXI y la universidad del futuro. In: MENEGHEL, Stela Maria; CAMARGO, Murilo Silva, SPELLER, Paulo (org.). De Havana a Córdoba: Duas Décadas de Educação Superior na AméricaLatina. Blumenau: Editora Nova Letra, 2018. p. 233-270.

BRANDENBURG, Uwe; DE WIT, Hans. The End of Internationalization. International Higher Education, 62, 2010

CANZANI, Álvaro Maglia; ARAUJO FILHO, Targino. Privatización, globalización mercantil y cooperación en educación superior en América Latina: la construcción de ENLACES. In: MENEGHEL, Stela Maria; CAMARGO, Murilo Silva, SPELLER, Paulo (org.). De Havana a Córdoba: Duas Décadas de Educação Superior na América-Latina. Blumenau: Editora Nova Letra, 2018. p. 152-182.

CAPES (Brasil). Edital no 41/2017 - Programa Institucional de Internacionalização Capes/PrInt. Disponible en: https://www.capes.gov.br/cooperacao-

internacional/multinacional/programa-institucional-de-internacionalizacao-capes-print. Acceso en: 20 abr. 2019.

DE WIT, Hans. Globalización e internacionalización de la educación superior. Revista de Universidad y Sociedad del Conocimiento (RUSC) v. 8, n. 2, p. 77-84, 2011.

DE WIT, Hans. Internationalization of higher education in the united states of america and europe: a historical, comparative, and conceptual analysis. Westport, CT: Greenwood Press, 2002.

FINARDI, Kyria Rebeca; AMORIM, Gabriel Brito; KAWACHI, Claudia. Internationalization and Language Assessment in Brazil: exploring the interface between language proficiency and rankings at UFES. Studies in English Language Teaching, v. 6, p. 139-154, 2018.

FINARDI, Kyria Rebeca; ARCHANJO, Renata. Reflections of Internationalization of Education in Brazil. In: INTERNATIONAL BUSINESS AND EDUCATION CONFERENCE, 2015, New York. Proceedings of... New York: Clute Institute, 2015. p. 504-510. v. 1. 
FINARDI, Kyria Rebeca; ARCHANJO, Renata. Washback effects of the Science without Borders, English without Borders and Languages without Borders programs in Brazilian language policies and rights. In: SIINER, Maarja; HULT, Francis M.; KUPISCH, Tanja (org.). Language policy and language acquisition planning. Cham: Springer, 2018. p. 173185 .

FINARDI, Kyria Rebeca. The slaughter of Kachru's five sacred cows in Brazil: affordances of the use of English as an international language. Studies in English Language Teaching, v. 2, n. 4, p. 401, 2014.

FINARDI, Kyria Rebeca. Language Policies and Internationalisation in Brazil: The Role(s) of English as an Additional Language. In: SCIRIHA, Lydia (org.). International Perspectives on Bilingualism, Newcastle upon Tyne, v. 1, p. 79-90, 2016a

FINARDI, Kyria Rebeca. Globalization an English in Brazil. In: FINARDI, Kyria Rebeca (org.). English in Brazil: views, policies and programs. Londrina: EDUEL, 2016b, v. 1, p. 15-36.

FINARDI, Kyria Rebeca. What Brazil can learn from Multilingual Switzerland and its use of English as a Multilingua Franca. Acta Scientiarum, Maringá, v. 39, p. 219-228, 2017a.

FINARDI, Kyria Rebeca. English as a global language in Brazil: A local contribution. In: GIMENEZ, Telma; EL KADRI, Michele Salles; CALVO, Luciana Cabrini Simões (org.). English as a lingua franca in teacher education: a Brazilian perspective. Berlin; Boston: De Gruyter Mouton, v. 1, p. 71-86. 2017b

FINARDI, Kyria Rebeca; CSILLAGH, Virag. Globalization and linguistic diversity in Switzerland: insights from the roles of national languages and English as a foreign language. In: GRUCZA, Sambor; OLPINSKA-SZKIELKO, Magdalena; ROMANOWSKI, Piotr. Advances in understanding multilingualism: a global perspective. Frankfurt am Main: Peter Lang GmbH, 2016.

FINARDI, Kyria Rebeca; FRANÇA, Claudio. O inglês na internacionalização da produção científica brasileira: evidências da subárea de linguagem e linguística. Intersecções, Jundiaí, v. 19, p. 234-250, 2016.

FINARDI, Kyria Rebeca; GUIMARÃES, Felipe Furtado. Internacionalização, rankings e publicações em inglês: a situação do Brasil na atualidade. Estudos em Avaliação Educacional, São Paulo, v.28, n.68, p.600-626, 2017.

FINARDI, Kyria Rebeca; LEAO, Roberta; PINHEIRO, Livia Melina. English in Brazil: Insights from the analysis of language policies, internationalization programs and the clil approach. Education and Linguistics Research, v. 2, p. 54-68, 2016.

FINARDI, Kyria Rebeca; ORTIZ, Ramón Andrés. Globalization, internationalization and education: what is the connection? IJAEDU- International E-Journal of Advances in Education, v. 1, p. 18-25, 2015. 
FINARDI, Kyria Rebeca; PORCINO, Maria Carolina. Tecnologia e Metodologia no Ensino de Inglês: Impactos da Globalização e da Internacionalização. Ilha do Desterro, v. 66, p. 239-284, 2014.

FINARDI, Kyria Rebeca; PORCINO, Maria Carolina. O papel do inglês na formação e na internacionalização da educação no Brasil. Horizontes de Linguística Aplicada, v. 14, n. 1, p. 109-134, 2015.

FINARDI, Kyria Rebeca; PREBIANCA, Gicele Vergine Vieira. Políticas linguísticas, internacionalização, novas tecnologias e formação docente: um estudo de caso sobre o curso de Letras Inglês em uma universidade federal. Leitura, Maceió, v. 1, p. 129-154, 2014.

FINARDI, Kyria Rebeca et al. Technology, English language teaching and internationalization at a crossroad: insights from the analysis of a virtual learning environment in Brazil. In: INTERNATIONAL CONFERENCE OF EDUCATION, RESEARCH AND INNOVATION, 2014, Sevilla. Proceedings of... Madrid: IATED, 2014. p. 1-12, v. 1.

FINARDI, Kyria Rebeca; SANTOS, Jane; GUIMARÃES, Felipe Furtado. A relação entre línguas estrangeiras e o processo de internacionalização: evidências da coordenação de letramento internacional de uma universidade federal. Interfaces Brasil/Canadá. Canoas, v.16, n.1, 2016.

FINARDI, Kyria Rebeca; TYLER, Jhamille. The role of English and technology in the internationalization of education: insights from the analysis of MOOCS. In:

INTERNATIONAL CONFERENCE ON EDUCATION AND NEW LEARNING TECHNOLOGIES, 7., 2015, Barcelona. Proceedings of ... Barcelona: IATED, 2015. p. 1118. v. 1.

GONZÁLEZ, Jorge. Internacionalización de la educación superior. Universidades, n. 33, 2007.

GUARGA, Rafael; SOSA, Fernando. La Conferencia Regional de Educación Superior y la Conferencia Mundial de 2009: el papel de América Latina. In: MENEGHEL, Stela Maria; CAMARGO, Murilo Silva, SPELLER, Paulo (org.). De Havana a Córdoba: duas décadas de educação superior na América-Latina. Blumenau: Editora Nova Letra, 2018. p. 139-151.

GUIMARAES, Felipe Furtado; FINARDI, Kyria Rebeca. Interculturalidade, Internacionalização E Intercompreensão: Qual A Relação? Ilha do Desterro, v. 71, p. 15-38, 2018.

GUIMARÃES, Felipe Furtado; FINARDI, Kyria Rebeca. Políticas Linguísticas para Internacionalização do Ensino Superior no Brasil: uma proposta. In: SILVA, Kleber (Org.). Linguística aplicada e seu compromisso com a sociedade. (En prensa).

HILDEBLANDO, Carlos Alberto; FINARDI, Kyria Rebeca. Internationalization and Virtual Collaboration: Insights from COIL Experiences. Ensino em Foco, v. 1, p. 19-33, 2018.

HUDZIK, John K. Comprehensive internationalization: from concept to action. Washington: NAFSA, 2011.

\begin{tabular}{l|l|l|l|l|l} 
(C) Rev. Inter. Educ. Sup. & Campinas, SP & v.6 & $1-23$ & e020031 & 2020
\end{tabular}


KAWACHI, Claudia; AMORIM, Gabriel Brito; FINARDI, Kyria Rebeca. The interface between the TOEFL ITP and internationalization and language assessment in Brazil. Studies in English Language Teaching, v. 5, p. 213-230, 2017.

KNIGHT, Jane. Updating the definition of internationalization. International Higher Education, n 33, 2003.

KNIGHT, Jane. Higher education in turmoil: the changing world of internationalization. Rotterdam: Sense Publishers, 2008.

LEITE, Denise; GENRO, Maria Elly Herz. Avaliação e internacionalização da educação superior: Quo vadis América Latina? Avaliação: Revista da Avaliação da Educação Superior, Campinas, v. 17, n. 3, p. 763-785, 2012.

LIMA, Manolita Correia; MARANHÃO, Carolina Machado Saraiva de Albuquerque. O sistema de educação superior mundial: entre a internacionalização ativa e passiva. Avaliação: Revista da Avaliação da Educação Superior, Campinas, v. 14, n. 3, p. 583-610, 2009.

LÓPEZ, Sara Fernández; SANMARTIN, Emilio Ruzo. Los procesos de internacionalización y globalización en la educación superior: un análisis de los países de la OCDE. Revista de Educación, v. 335, p. 385-413, 2004.

MAUÉS, Olgaíses Cabral; SOUZA, Michele Borges. A transnacionalização e a expansão da educação superior. Educação em Questão, v. 56, n. 47, p. 151-173, 2018.

MINTO, Lalo Watanabe. Educação superior no Brasil e a agenda ultraconservadora. Presentación en la clase inaugural del Programa de Posgrado en Educación (PPGE) en la Universidad Federal del Espírito Santo (UFES), en 1 de abril de 2019.

MOROSINI, Marilia Costa; CORTE, Marilene Gabriel Dalla. Teses e realidades no contexto da internacionalização da educação superior no Brasil. Educação em Questão, v. 56, n. 47, p. 97-120, 2018.

NILSSON, Bengt. Internationalisation at home from a Swedish perspective: the case of Malmö. Journal of Studies in International Education, v.7, n.1, 2003.

ORTIZ, Ramón Andrés; FINARDI, Kyria Rebeca. Social inclusion and CLIL: evidence from La Roseraie. In: INTERNATIONAL CONFERENCE ON EDUCATION, RESEARCH AND INNOVATION, 2015, Sevilla. Proceedings of... Madrid: Iated, 2015. p. 7660-7666. v. 1.

PATEL, Fay; LYNCH, Hayley. Glocalization as an Alternative to Internationalization in Higher Education: Embedding Positive Glocal Learning Perspectives. International Journal of Teaching and Learning in Higher Education, v. 25, n. 2, p. 223-230, 2013.

PICCIN, Gabriela Freire Oliveira; FINARDI, Kyria Rebeca. A internacionalização a partir de diferentes loci de enunciação: as concepções de sujeitos praticantes do currículo profissional. Trabalhos em Linguística Aplicada, Campinas, v.58, p.313-340, 2019. Disponível em: Aceso en: https://periodicos.sbu.unicamp.br/ojs/index.php/tla/article/view/8653317. Acesso en: 25 abr. 2019.

\begin{tabular}{l|c|c|c|c|c} 
(C) Rev. Inter. Educ. Sup. & Campinas, SP & v.6 & $1-23$ & $\mathrm{e} 020031$ & 2020 \\
\hline
\end{tabular}


PORCINO, Maria Carolina; FINARDI, Kyria Rebeca. Globalization and internationalization in ELT: methodology, technology and language policy at a crossword in Brazil. In:

International Conference of Education, Research and Innovation, 2014, Sevilla. ICERI2014 Proceedings. Madrid: IATED, 2014. v. 1. p. 1-11.

RAJAGOPALAN, Kanavillil. Por uma linguística crítica: linguagem, identidade e a questão ética. São Paulo: Parábola Editorial, 2003.

SÁNCHEZ-MECA, Julio. Cómo realizar una revisión sistemática y un meta-análisis. Aula Abierta, v. 38, n. 2, p. 53-64, 2010.

SÁNCHEZ-MECA, Julio; BOTELLA, Juan. Revisiones sistemáticas y meta-análisis: herramientas para la práctica profesional. Papeles del Psicólogo, v. 31, n. 1, p. 7-17, 2010.

SANTOS, Boaventura de Sousa. A crítica da razão indolente: contra o desperdício da experiência. In: SANTOS, Boaventura de Sousa. Para um novo senso comum: a ciência, o direito e a política na transição paradigmática. São Paulo: Cortez, 2009.

SANTOS, Boaventura de Sousa. A universidade no século XXI: para uma reforma democrática e emancipatória da universidade. São Paulo: Cortez, 2011, 3 ed.

TAQUINI, Renini; FINARDI, Kyria Rebeca.; AMORIM, Gabriel Brito. English as a Medium of Instruction at Turkish State Universities. Education and Linguistics Research, v. 3, p. 35-53, 2017.

UNITED NATIONS. Human Development Report 1999. United Nations Development Programme. Oxford: Oxford University Press, 1999.

VAVRUS, Francis; PEKOL, Amy. Critical Internationalization: Moving from theory to Practice. FIRE: Forum for International Research in Education, v. 2, n. 2, 2015.

VIEIRA, Gicele Vergine.; FINARDI, Kyria Rebeca.; PICCIN, Gabriela Freire Oliveira. Internacionalizando-Se: Os Desafios para os Institutos Federais de Educação, Ciência e Tecnologia do Brasil. Revista Ibero-Americana de Estudos em Educação, v. 13, p. 394410, 2018.

Agradecimento a Fapes. 